\title{
SUMS OF REAL PARTS OF EIGENVALUES OF PERTURBED MATRICES
}

\author{
M. I. GIL'
}

Abstract. Let $A$ be $\tilde{A}$ be $n \times n$ matrices, whose eigenvalues are $\lambda_{k}$ and $\tilde{\lambda}_{k}$, respectively. Assuming that $A$ is Hermitian, we prove the inequality

$$
\left[\sum_{k=1}^{n}\left|\operatorname{Re} \tilde{\lambda}_{k}-\lambda_{k}\right|^{p}\right]^{1 / p} \leqslant N_{p}\left(E_{R}\right)+\tilde{b}_{p} N_{p}\left(E_{I}\right) \quad(2 \leqslant p<\infty)
$$

where $N_{p}(A)$ is the Schatten-von Neumann norm of $A, E=\tilde{A}-A, E_{R}=\left(E+E^{*}\right) / 2, E_{I}=$ $\left(E-E^{*}\right) / 2 i$, and $\tilde{b}_{p} \leqslant p e^{1 / 3}$. That inequality is generalized then to the Schatten-von Neumann operators.

Mathematics subject classification (2010): 15A42, 15A18, 47A10, 47B10.

Keywords and phrases: Matrices, inequalities for eigenvalues, Schatten-von Neumann ideals.

\section{REFERENCES}

[1] N. Dunford, And J.T. Schwartz, Linear Operators, part II. Spectral Theory, Interscience Publishers, New York, London, 1963.

[2] M.I. GIL', Operator Functions and Localization of Spectra, Lecture Notes In Mathematics vol. 1830, Springer-Verlag, Berlin, 2003.

[3] M.I. GIL', Lower bounds for eigenvalues of Schatten-von Neumann operators, J. Inequal. Pure Appl. Mathem., 8, 3 (2007), 117-122.

[4] I.C. Gohberg, And M.G. KRein, Introduction to the Theory of Linear Nonselfadjoint Operators, Trans. Mathem. Monographs, v. 18, Amer. Math. Soc., Providence, R. I., 1969.

[5] I.C. Gohberg, And M.G. Krein, Theory and Applications of Volterra Operators in Hilbert Space, Trans. Mathem. Monogr., Vol. 24, Amer. Math. Soc., R. I. 1970.

[6] A.J. HofFMAN, AND H.W. WIELlandT, The variation of the spectrum a normal matrix, Duke Math. J., 20 (1953), 37-39.

[7] W. Kahan, Spectra of nearly Hermitian matrices, Proc. Am. Math. Soc., 48 (1975), 11-17.

[8] T. KATO, Perturbation Theory for Linear Operators, Springer, Berlin, 1966.

[9] L. MiRs KY, Symmetric gage functions and unitarily invariant norms, Q. J. Math., 11 (1960), 50-59.

[10] M. SigG, A Minkowski-type inequality for the Schatten norm, J. Inequal. Pure Appl. Math., 6, 3 (2005), Paper No. 87, 7 p., electronic only.

[11] G. W. Stewart and Ji-Guang Sun, Matrix Perturbation Theory, Academic Press, New York, 1990. 